\title{
Natural Infection of Southern Highbush Blueberry (Vaccinium corymbosum Interspecific Hybrids) by Xylella fastidiosa subsp. fastidiosa
}

\author{
Dario Di Genova, ${ }^{1,2}$ Kippy J. Lewis, ${ }^{3}$ and Jonathan E. Oliver ${ }^{3, \dagger}$ \\ ${ }^{1}$ Department of Crop and Soil Sciences, University of Georgia, Tifton Campus, Tifton, GA 31793, U.S.A. \\ ${ }^{2}$ Department of Agronomy, Food, Natural Resources, Animals and Environment, University of Padova, 35020 Legnaro, Italy \\ ${ }^{3}$ Department of Plant Pathology, University of Georgia, Tifton Campus, Tifton, GA 31793, U.S.A.
}

\begin{abstract}
$X y l e l l a$ fastidiosa $(X f)$ is an emerging insect-vectored, xylem-limited bacterium that can cause disease on several economically important fruit and tree crops including almond, blueberry, citrus, grapevine, peach, and pecan. On blueberry, $X f$ causes bacterial leaf scorch (BLS), which is prevalent in the southeastern United States. This disease, previously reported to be caused by $X f$ subsp. multiplex $(X f m)$, can result in rapid plant decline and death of southern highbush (SHB) blueberry cultivars. In 2017, a survey of blueberry plantings in southern Georgia (U.S.A.) confirmed the presence of $X f$-infected plants in eight of nine sites examined, and seven isolates were cultured from infected plants. Genetic characterization of these isolates through single-locus and multilocus sequence analysis revealed that three isolates from two sites belonged to $X f$ subsp. fastidiosa $(X f f)$, with significant similarity to isolates from grapevine. After these three isolates were artificially inoculated onto greenhouse-grown SHB blueberries (cv. 'Rebel'), symptoms typical of BLS developed, and Xff

infection was confirmed through genetic characterization and reisolation of the bacterium to fulfill Koch's postulates. Because all previously reported $X f$ isolates from blueberry have been characterized as $X f m$, this is the first time that isolation of $X f f$ has been reported from naturally infected blueberry plantings. The potential impact of $X f f$ isolates on disease management in blueberry requires further exploration. Furthermore, given that isolates from both $X f m$ and $X f f$ were obtained within a single naturally infected blueberry planting, blueberry in southern Georgia may provide opportunities for intersubspecific recombination between $X f f$ and $X f m$ isolates.

Keywords: Xylella fastidiosa subsp. fastidiosa, Xylella fastidiosa subsp. multiplex, bacterial leaf scorch, genetic variability, blueberry diseases, southern highbush blueberry, multilocus sequence analysis, Koch's postulates
\end{abstract}

Xylella fastidiosa $(X f)$ is a xylem-inhabiting, gram negative, fastidious, plant pathogenic bacterium that infects a wide variety of host plants. In recent years, this pathogen has emerged as a considerable issue on an increasing number of perennial crops and trees (Almeida and Nunney 2015; Almeida et al. 2019; Chatterjee et al. 2008). Economically important agricultural crops that are affected by $X f$ include almond, citrus, coffee, grapevine, peach, pecan, plum, and, more recently, blueberry and olive, as well as several shade trees and ornamentals such as elm, maple, oak, oleander, and sycamore (Chang et al. 2009; Chatterjee et al. 2008; Hopkins and Purcell 2002; Loconsole et al. 2014; Purcell 2013). In addition, many wild plants (wild grasses, sedges, vines, and trees) are known to act as natural reservoirs and may carry the pathogen often without symptoms (Janse and Obradovic 2010; Purcell and Saunders 1999). Transmission of $X f$ is mediated by xylem sap-feeding vectors, including leafhoppers, sharpshooters (family Cicadellidae), and spittlebugs (family Cercopoidea), in a complex biological and ecological plant-vector-pathogen interaction that involves bacterial retention and multiplication within the vector's foregut and subsequent dispersal to another plant during xylem feeding (Brlansky et al. 1983; Purcell and Finlay 1979; Purcell et al. 1979).

${ }^{\dagger}$ Corresponding author: J. E. Oliver; jonathanoliver@uga.edu

Funding: This research was partially supported by the USDA National Institute of Food and Agriculture, HATCH project no. 1016575, and a grant jointly funded by the University of Georgia College of Agricultural and Environmental Sciences, Office of Research, and Department of Plant Pathology.

*The $\boldsymbol{e}$-Xtra logo stands for "electronic extra" and indicates that one supplementary figure and two supplementary tables are published online.

The author(s) declare no conflict of interest.

Accepted for publication 15 April 2020.

(C) 2020 The American Phytopathological Society
Bacterial leaf scorch (BLS) of blueberry was first observed in 2004 and later confirmed to be caused by $X f$ in the blueberry production region of southern Georgia (Chang et al. 2009) and Florida (Harmon and Hopkins 2009). Disease symptoms include marginal chlorosis and subsequent scorching (necrotic burns) of leaves, premature defoliation, and dieback, ultimately leading to death of the entire blueberry bush (Brannen et al. 2016; Chang et al. 2009). Xf has been observed to most severely affect southern highbush (SHB) blueberry (Vaccinium corymbosum interspecific hybrids), with varying disease incidence and severity across different cultivars (Brannen et al. 2008); however, rabbiteye blueberries ( $V$. ashei) can also be infected and show symptoms in some cases (Ferguson et al. 2017). Several sharpshooter leafhoppers are believed to vector $X f$ in blueberry plantings in the southeastern United States, with the glassy-winged sharpshooter (Homalodisca vitripennis) being the most frequently observed (Tertuliano et al. 2012).

Based upon genetic characterization of the 16S-23S intergenic spacer region and multilocus sequence typing analyses (MLSTs), the species $X f$ has been shown to be composed of multiple genetically distinct subspecies (Nunney et al. 2014; Parker et al. 2012; Scally et al. 2005; Schaad et al. 2004). These subspecies include $X f$ subsp. fastidiosa (Xff), isolated from alfalfa, almond, grape, and maple; $X f$ subsp. pauca $(X f p)$, isolated from citrus, coffee, and olive; $X f$ subsp. sandyi, isolated from oleander; and $X f$ subsp. multiplex $(X f m)$, isolated from various hosts including almond, blueberry, peach, pecan, plum, and several shade trees. Genetic differences between and within subspecies are assumed to determine the host range of individual isolates; however, the exact genetic determinants of $X f$ pathogenicity on certain hosts are not known. Cross-inoculations with isolates from different subspecies have shown that Xff isolates from grapevine and alfalfa do not infect peach or citrus, and $X f m$ isolates from peach do not infect grapevine or citrus (Hopkins and Purcell 2002; Janse and Obradovic 2010). Likewise, Xfp isolates from South American citrus do not seem to colonize coffee and vice versa (Almeida et al. 2008; Nunney et al. 2013). Furthermore, host range tests with Xfm isolates from tree species recently showed that each $X f$ strain had a unique host range (Nunney et al. 2019). By contrast, 
some plant hosts can be naturally infected by multiple subspecies of $X f$, because isolates from both Xfm and Xff have been found causing almond leaf scorch (Almeida and Purcell 2003). Despite the documented host specificity differences between isolates, the limited variation within genes used in $X f$ phylogenetic studies (e.g., MLST) is unlikely to explain the differences in biological traits and infectivity among $X f$ isolates (Almeida and Nunney 2015; Killiny and Almeida 2011; Parker et al. 2012).

Even though BLS of blueberry is known to cause severe, widespread yield reductions in the southeastern U.S. blueberry production region within Georgia and Florida, only a relatively small number of $X f$ isolates have been cultured from in-field symptomatic blueberry plants and characterized genetically. Among the reported blueberry $X f$ isolates characterized to date (Hopkins et al. 2012; Nunney et al. 2014; Oliver et al. 2014, 2015; Parker et al. 2012), all have been reported to belong to a single clade of $X \mathrm{fm}$ consisting of intersubspecific recombinants (Nunney et al. 2014). Nonetheless, $X f f$ is prevalent (likely endemic) in the southeastern United States, being isolated from grapes (Vitis sp.) and muscadines (Vitis rotundifolia) as well as elderberry and lupine (Hopkins and Purcell 2002; Parker et al. 2012), and artificial inoculation tests on SHB blueberry with several different $X f f$ and $X f m$ isolates have shown the capability of both subspecies to cause leaf scorching symptoms in controlled greenhouse conditions (Oliver et al. 2015).

The objective of this study was to better understand the distribution and diversity of $X f$ in blueberry plantings in southern Georgia. To that end, we surveyed nine blueberry plantings in southern Georgia and attempted to culture the pathogen from infected blueberry plants. Obtained isolates were initially genetically characterized using the $r p o D$ gene. This was followed by more extensive characterization using two different multilocus sequence typing approaches. Based upon our findings, we carried out Koch's postulates with some of the isolates obtained. Our results show that isolates from both $X \mathrm{fm}$ and $X f f$ are present in naturally infected blueberry plantings in southern Georgia, suggesting that SHB blueberry may be a permissive host for multiple $X f$ subspecies and accordingly provide opportunities for intersubspecific recombination and the emergence of new virulent $X f$ strains. Furthermore, these findings have the potential to impact management of $X f$ in blueberry production fields in the southeastern United States.

\section{Materials and Methods}

Field survey and isolation of $\boldsymbol{X} \boldsymbol{f}$. In September 2017, plantings of SHB blueberry in three adjacent counties in southeastern Georgia were surveyed for the presence of $X f$. In total, nine sites were surveyed including three sites each in Bacon, Pierce, and Ware counties in Georgia. Surveyed sites in Bacon County were located at least 18 and 25 miles from the nearest sites in Pierce and Ware counties, respectively, whereas sites in Pierce County were located at least 20 miles from the nearest site in Ware County. Within each county, the three surveyed sites were located approximately 6 to 12 miles apart in Bacon County, 6 to 8 miles apart in Pierce County, and 2 to 7 miles apart in Ware County. Plantings in Ware and Pierce counties had been established between 3 and 6 years prior to the survey, and plantings in Bacon County ranged from 3 to 10 years of age. The original sources of surveyed plants were not available for all plants and sites, but plants originated from multiple nurseries within Florida and Georgia or were propagated on-farm. At each of the nine sites, leaf samples were collected from at least five plants per site showing typical BLS symptoms (Fig. 1). Total DNA was extracted from three to five midribs and petioles of the collected leaf samples using a modified cetyltrimethylammonium bromide (CTAB) protocol (Doyle and Doyle 1987). The presence of $X f$ was initially verified by polymerase chain reaction (PCR) testing using the RST31/33 primer pair (Minsavage et al. 1994) and subsequently confirmed using the HL5/6 primer pair (Francis et al. 2006). PCR was carried out using a S1000 Thermal Cycler (Bio-Rad, Hercules, CA) with a GoTaq (Promega, Fitchburg, WI) PCR kit, with the following reagents: $10 \mu \mathrm{l}$ of $2 \times$ GoTaq Green Master Mix, $2 \mu$ l each of forward and reverse primers (10 $\mu \mathrm{M}$ for HL5/6 and $100 \mu \mathrm{M}$ for RST31/33), $1 \mu \mathrm{l}$ of DNA (1 to
$3 \mathrm{ng} / \mu \mathrm{l}$ ), and $5 \mu \mathrm{l}$ of $\mathrm{dH}_{2} \mathrm{O}$. PCR conditions were as follows: $5 \mathrm{~min}$ at $94^{\circ} \mathrm{C}$ followed by 34 cycles of $94^{\circ} \mathrm{C}$ for $30 \mathrm{~s}, 55^{\circ} \mathrm{C}$ for 30 $\mathrm{s}$, and $72^{\circ} \mathrm{C}$ for $45 \mathrm{~s}$, and concluding with $72^{\circ} \mathrm{C}$ for $2 \mathrm{~min}$. Appropriate positive and negative controls were included, and amplified products were visualized by gel electrophoresis on $1 \%$ agarose gels (1× TAE buffer) stained with GelRed Nucleic Acid Stain (Biotium, Fremont, CA) using a Gel Doc XR+ (Bio-Rad) gel imager.

After confirmation of $X f$ infection via PCR, attempts were made to isolate $X f$ from the midribs and petioles of symptomatic blueberry leaf tissue. $X f$ was isolated on periwinkle wilt (PW) and $\mathrm{PW}+$ media according to the protocol previously described by Davis et al. (1981). Following DNA extractions from bacterial biomass via a modified CTAB protocol (Doyle and Doyle 1987), isolates were confirmed as $X f$ by PCR using the RST31/33 primer pair as described above. Glycerol stocks were prepared by scraping $X f$ isolates from plates, suspending them in a PD2 and 20\% glycerol solution (Davis et al. 1980), and placing them at $-80^{\circ} \mathrm{C}$ for long-term storage.

Genetic characterization of $\boldsymbol{X} \boldsymbol{f}$ isolates using $\boldsymbol{r p o D}$. RST31/33 primer pair amplicons from PCR on total DNA extracted from symptomatic plant samples were purified using the E.Z.N.A. Cycle Pure Kit (Omega Bio-Tek, Norcross, GA) and sequenced in both directions via Sanger sequencing by Eurofins Genomics (Louisville, $\mathrm{KY}$ ). These primers amplify a portion of the RNA polymerase sigma-70 factor $r p o D$ locus from $X f$. Following isolation of the bacteria from symptomatic plant material, RST31/33 primer pair PCR amplicons (733 nt) were also sequenced in both directions from each of the bacterial isolates. Returned sequences for each amplicon were reviewed for signal quality on FinchTV 1.5.0 software (Geospiza) and then imported into SeaView 4.6.4 software (Galtier et al. 1996; Gouy et al. 2010) for manual editing and alignment. Obtained sequences were analyzed and aligned alongside additional rpoD $X f$ sequences imported from the GenBank database (https:// www.ncbi.nlm.nih.gov/genbank/) in July 2018 (Supplemental Table S1). Phylogenetic trees were generated to compare all sequences using the neighbor-joining (NJ) method in MEGA7 (Kumar et al. 2016; Saitou and Nei 1987).

Genetic characterization of $X f$ isolates using multilocus sequence analysis of environmentally mediated genes (MLSA-E). For additional genetic characterization of $X f$ isolates, PCR was carried out to amplify bacterial DNA using primers (Table 1) for amplifying the nine "environmentally mediated" genes responsible for host infection and virulence regulation described by Parker et al. (2012). Amplified products were visualized by agarose gel electrophoresis, purified, prepared, sequenced, and analyzed as described for the $r p o D$ genetic characterization above. Nine phylogenetic trees (one for each gene) were generated using the NJ method in MEGA7. Finally, a concatenated nine-gene sequence for each $X f$ isolate was assembled to allow for multilocus alignment. Concatenated isolate sequences obtained in this study, as well as additional $X f$ sequences imported from the GenBank database (https://www.ncbi.nlm.nih. gov/genbank/) in July 2018 (Supplemental Table S2), were used to generate a single master phylogenetic tree using the NJ method in MEGA7.

Genetic characterization of $\boldsymbol{X} \boldsymbol{f}$ isolates using MLST genes. For the three isolates obtained from a single field site (AlmaReb1, AlmaReb2, and AlmaReb3), additional genetic characterization was carried out using the MLST approach reported previously by Scally et al. (2005) and Yuan et al. (2010). Primers were used to amplify seven additional $X f$ genes (Table 1) via PCR as described above. Amplified products were visualized by agarose gel electrophoresis, purified, prepared, sequenced, and analyzed as described for the $r p o D$ genetic characterization above. To determine MLST sequence types for each isolate, obtained sequences were compared with the $X f$ MLST sequence database (https://pubmlst.org/xfastidiosa) hosted at the University of Oxford (Jolley et al. 2004; Scally et al. 2005).

Inoculation of blueberry with $X f f$ isolates. Three isolates (AlmaReb1, AlmaReb2, and PierceMed1) obtained from blueberry and identified as belonging to Xff based upon phylogenetic analyses were used to inoculate SHB blueberries in the greenhouse. Blueberry plants originally grown from tissue culture (cv. 'Rebel' [PPA18, 
138]) (NeSmith 2008) were obtained from Agri-Starts (Apopka, FL) and transplanted into a one-part sand, three-part pine bark mulch mixture in 9.5 -liter and $25.7-\mathrm{cm}$ (diameter) pots. Plants were maintained in the greenhouse at 28 to $35^{\circ} \mathrm{C}$ under natural sunlight and watered as needed. Plants were fertilized twice, the first time 15 days after potting and the second time 3 months later, with a granular slow-release fertilizer (Osmocote Smart Release Plant Food Plus Outdoor and Indoor 15-9-12, The Scotts Company, Marysville, $\mathrm{OH})$ at the manufacturer's recommended rate for indoor use. Between 1 and 2 months after initial transplanting, blueberry plants were inoculated with Xff isolates using a 1-ml tuberculin syringe according to the protocol described previously (Chang et al. 2009; Oliver et al. 2015). Xff isolates were grown out from previously prepared glycerol stocks on PW medium for 10 days at $28^{\circ} \mathrm{C}$ and then suspended in succinate-citrate-phosphate (SCP) buffer prior to inoculation (De La Fuente et al. 2013). In total, each Xff isolate was used to inoculate three blueberry plants, and three plants were inoculated with SCP buffer only. Two weeks after the original inoculations, all plants were reinoculated in the same manner to ensure infection.

PCR confirmation of greenhouse blueberry infection and reisolation from symptomatic plants. At 120 days postinoculation, three lower leaves were collected from the originally inoculated stems of all plants and tested for the presence of $X f$ by PCR using the RST31/33 primer pair. At 150 days postinoculation, leaf samples were collected from $X f$-infected plants, and attempts were made to reisolate the bacterium using the protocol described previously for field sample isolations (Davis et al. 1981). Bacterial colonies obtained were purified on PW medium, and total DNA was extracted using the modified CTAB protocol described previously to allow for PCR confirmation that the obtained isolates were $X f$. PCR confirmation was carried out using primer pairs RST31/33 and copB-F/R (Table 1), and amplicons were sequenced via Sanger sequencing by Eurofins Genomics to verify that the cultured bacteria matched the identity of the isolate originally used to inoculate each blueberry plant, respectively.

\section{Results}

Field survey and $X \boldsymbol{f}$ isolation results. A total of 47 leaf samples of SHB blueberry from nine field sites with evident BLS symptoms (Fig. 1) were collected and subsequently tested for the presence of $X f$ using PCR. PCR testing of leaf tissue indicated $X f$-positive samples from 8 of $9(88.8 \%)$ blueberry sites and 22 of $47(47 \%)$ collected
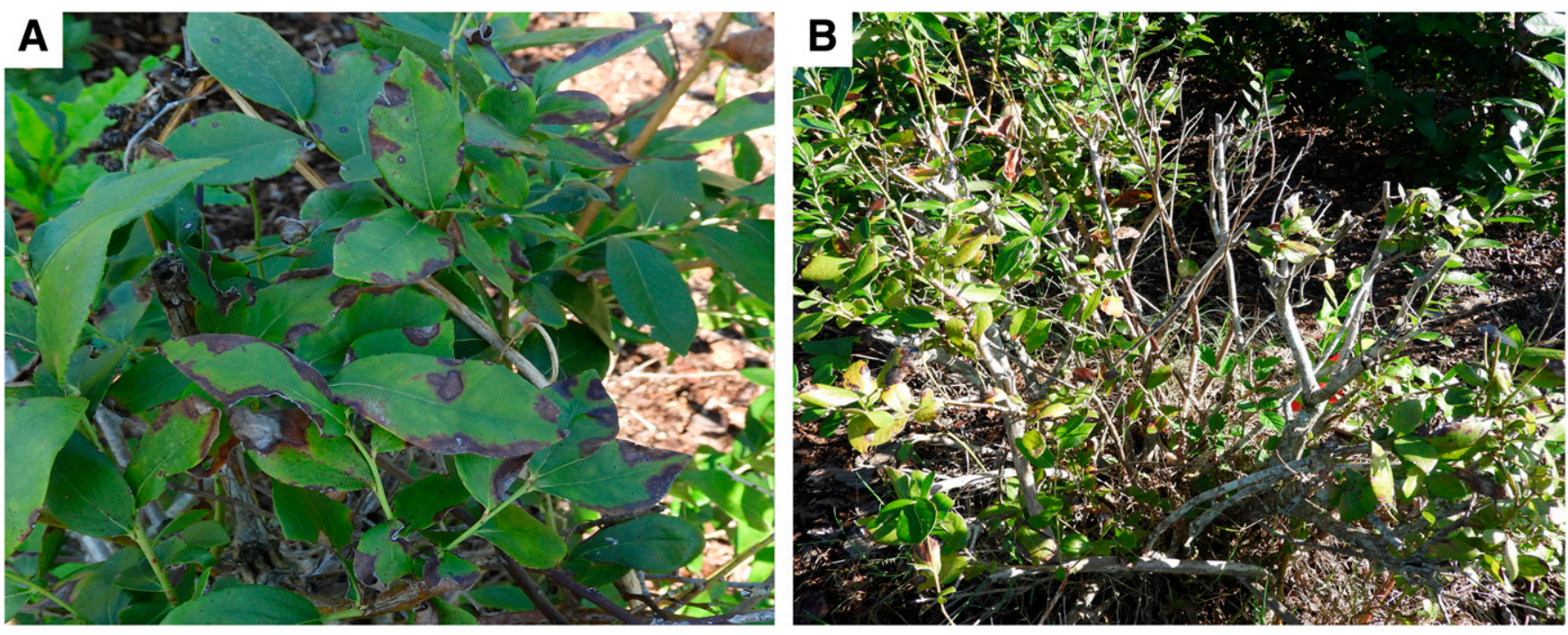

Fig. 1. Bacterial leaf scorch symptoms observed on blueberry during field survey: A, leaf scorch, and B, defoliation and dieback.

Table 1. Primers used for PCR amplification and sequencing of genes characterized in this study ${ }^{\mathrm{a}}$

\begin{tabular}{|c|c|c|c|c|}
\hline Gene & Role in study & Primers (F/R) & Amplicon size (bp) & Reference \\
\hline & Pathogen detection & HL5/6 & & Francis et al. (2006) \\
\hline rpoD & Pathogen detection and genetic characterization & RST-31/RST-33 & 733 & Minsavage et al. (1994) \\
\hline$a c v B$ & Genetic characterization (MLSA-E) & acvB-F/acvB-R & 743 & Parker et al. (2012) \\
\hline $\operatorname{cop} B$ & Genetic characterization (MLSA-E) & copB-F/copB-R & $607-862$ & Parker et al. (2012) \\
\hline$c v a C$ & Genetic characterization (MLSA-E) & cvaC-F/cvaC-R & 330 & Parker et al. (2012) \\
\hline $\operatorname{fim} A$ & Genetic characterization (MLSA-E) & fimA-F/fimA-R & 557 & Parker et al. (2012) \\
\hline$g a a$ & Genetic characterization (MLSA-E) & gaa-F/gaa-R & 1,129 & Parker et al. (2012) \\
\hline pglA & Genetic characterization (MLSA-E) & pglA-F/pglA-R & $828 / 829$ & Parker et al. (2012) \\
\hline pilA & Genetic characterization (MLSA-E) & pilA-F/pilA-R & 405 & Parker et al. (2012) \\
\hline$r p f F$ & Genetic characterization (MLSA-E) & rpfF-F/rpfF-R & 825 & Parker et al. (2012) \\
\hline$x a d A$ & Genetic characterization (MLSA-E) & xadA-F/xadA-R & $1,087 / 1,108$ & Parker et al. (2012) \\
\hline cysG & Genetic characterization (MLST) & cysG-F/cysG-R & 600 & Scally et al. (2005) \\
\hline $\operatorname{glt} T$ & Genetic characterization (MLST) & gltT-F/gltT-R & 654 & Scally et al. (2005) \\
\hline holC & Genetic characterization (MLST) & holC-F/holC-R & 379 & Scally et al. (2005) \\
\hline leuA & Genetic characterization (MLST) & leuA-F/leuA-R & 708 & Scally et al. (2005) \\
\hline malF & Genetic characterization (MLST) & malF-F/malF-R & 730 & Scally et al. (2005) \\
\hline пиоL & Genetic characterization (MLST) & nuoL-F/nuoL-R & 557 & Scally et al. (2005) \\
\hline petC & Genetic characterization (MLST) & petC-F/petC-R & 533 & Scally et al. (2005) \\
\hline
\end{tabular}

${ }^{a}$ MLSA-E = multilocus sequence analysis of environmentally mediated genes, and MLST = multilocus sequence typing. 
samples (Table 2). The only site from which $X f$-positive leaf material was not confirmed was site 2 in Pierce County, Georgia. Attempts to isolate the $X f$ bacterium from the collected samples resulted in the isolation of seven $X f$ isolates from blueberry. Three of these isolates (AlmaReb1, AlmaReb2, and AlmaReb3) came from SHB cultivar 'Rebel' at site 7 in Bacon County, three additional isolates (AlmaStar1, AlmaStar2, AlmaStar3) came from cultivar 'Star' at site 9 in Bacon County, and the seventh isolate (PierceMed1) came from cultivar 'Meadowlark' at site 1 in Pierce County (Table 3).

Genetic characterization of $\boldsymbol{X f}$ isolates using $\boldsymbol{r p o D}$. For the seven isolates obtained from blueberry, sequencing of the $r p o D$ gene fragment unexpectedly revealed two groups of sequences: group 1 (consisting of four isolates with identical sequences: AlmaReb3, AlmaStar1, AlmaStar2, and AlmaStar3) and group 2 (consisting of three isolates with identical sequences: AlmaReb1, AlmaReb2, and PierceMed1). BLAST analysis of the resulting sequences versus $X f$ sequences in the GenBank nucleotide collection showed the most similarity (99.4\%) between group 1 isolates and $\mathrm{Xfm}$ isolate M12 (CP000941) from almond, whereas group 2 isolates showed $100 \%$ identity with Xff isolate Temecula1 (AE009442) from grapevine. By contrast, the $r p o D$ fragments sequenced for the group 1 isolates showed $98.6 \%$ identity to the sequences from group 2 isolates. Sequences for the rpoD amplicon generated from the seven isolates in this study were deposited in GenBank as accession numbers MN590433 to MN590439. A phylogenetic tree produced using rpoD sequences (Fig. 2) showed three predominant clades: one consisting of isolates identified as Xfp from coffee, citrus, and other hosts; one consisting of isolates from grapevine and muscadines identified as $X f f$; and one consisting of isolates from blueberry, almond, and shade trees identified as $X \mathrm{fm}$. The group 1 isolates from this study clustered with $X f m$ sequences from blueberry, whereas the group 2 isolates from this study clustered with $X f f$ isolates from grapevine. Sequencing of the rpoD fragments amplified from the DNA samples extracted from the field plants (out of which the isolates were cultured originally) produced the same sequence result as the PCR using DNA from the isolated bacteria. From site 7 in Bacon County, where three isolates were obtained, $r p o D$ sequences from the five $X f$-positive plants resulted in sequences from two plants that matched group 2 sequences and three that matched group 1 .

Genetic characterization of $\boldsymbol{X} \boldsymbol{f}$ isolates using MLSA-E. Sequences obtained from the seven isolates in this study for each of the nine MLSA-E genes were deposited in GenBank as accession numbers MN590440 to MN590502. MLSA-E analysis also supported the results from $r p o D$ in that the seven cultured $X f$ isolates grouped into two main groups (group 1 and group 2). The pattern observed with the $r p o D$ sequences (sequences were identical within each of the two groups) was consistent across eight of the nine sequenced MLSA-E genes. For the $\operatorname{cop} B$ gene fragment sequence, isolate AlmaReb2 was found to have an extra $15 \mathrm{nt}$ starting at position 104 within the $\operatorname{cop} B$ amplicon compared with the other group 2 isolates, AlmaReb1 and PierceMed1. Further comparisons with available GenBank sequences for $\operatorname{cop} B$ proved AlmaReb2 to be a new haplotype, different from any $c o p B$ sequence previously obtained

Table 2. Xylella fastidiosa (Xf) PCR testing results from the 2017 Georgia southern highbush (SHB) blueberry survey

\begin{tabular}{lllc}
\hline $\begin{array}{l}\text { Site } \\
\text { ID }\end{array}$ & $\begin{array}{c}\text { Georgia } \\
\text { county }\end{array}$ & \multicolumn{1}{c}{ SHB cultivars } & $\begin{array}{c}\boldsymbol{X} \boldsymbol{f} \text { positives/total } \\
(\boldsymbol{\%})\end{array}$ \\
\hline 1 & Pierce & Meadowlark, Star, Farthing & $3 / 5(60 \%)$ \\
2 & Pierce & Star & $0 / 5(0 \%)$ \\
3 & Pierce & Meadowlark & $1 / 5(20 \%)$ \\
4 & Ware & Rebel & $1 / 5(20 \%)$ \\
5 & Ware & Star, Rebel & $3 / 5(60 \%)$ \\
6 & Ware & Rebel, Sweet Crisp, Farthing & $3 / 7(43 \%)$ \\
7 & Bacon & Rebel & $5 / 5(100 \%)$ \\
8 & Bacon & FL 86-19(V1) & $1 / 5(20 \%)$ \\
9 & Bacon & Star & $5 / 5(100 \%)$ \\
& & & Total 22/47 (47\%) \\
\hline
\end{tabular}

for $X f$. In addition, once the concatenated sequences of all nine MLSA-E genes were examined, it was apparent that AlmaReb1 and PierceMed1 also represented new haplotypes as well, with a unique combination of sequences from $\operatorname{cop} B$ and $x a d A$, although neither the $\operatorname{cop} B$ nor $x a d A$ sequences obtained for these isolates was unique on its own.

Single-gene MLSA-E phylogenetic trees consistently showed that sequences from group 1 isolates grouped with $X \mathrm{fm}$ sequences and group 2 isolates with Xff for each of the nine genes (data not shown). Furthermore, the master MLSA-E tree supported the classification of group 1 isolates as Xfm and group 2 isolates as Xff (Fig. 3). Group 2 isolates grouped most closely with haplotype GR_GAFL from Parker et al. (2012), consisting of Florida and Georgia muscadine and grapevine isolates. Group 1 isolates were identical to Parker et al. (2012) haplotype BLUEBERRY3.

Genetic characterization of $\boldsymbol{X} \boldsymbol{f}$ isolates using MLST. Sequences obtained from isolates AlmaReb1, AlmaReb2, and AlmaReb3 for each of the seven MLST genes were deposited in GenBank as accession numbers MN590503 to MN590523. MLST gene sequences (Table 4) indicated that the group 1 isolate AlmaReb3 was identical to Sequence Type 42 (ST42), which includes three Xfm isolates previously sequenced from blueberry and two $X \mathrm{fm}$ isolates from giant ragweed and one from western soapberry (Nunney et al. 2013). Group 2 isolates AlmaReb1 and AlmaReb2 were identical to ST1 (Table 4), which includes, among others, the Xff type isolate from grapevine Temecula1.

Inoculation of blueberry with $\boldsymbol{X} \boldsymbol{f}$ and fulfillment of Koch's postulates. Greenhouse-grown blueberry plants inoculated with $X f f$ isolates AlmaReb1, AlmaReb2, and PierceMed1 developed typical BLS symptoms after 7 weeks postinoculation (Supplemental Figure S1). Plant inoculation with each of the isolates, respectively, caused definitive symptoms. Somewhat more severe symptoms were observed in the plants inoculated with isolate AlmaReb2 compared with the plants inoculated with the other two Xff isolates tested. PCR testing of leaves indicated $X f$ infection of all $X f$-inoculated plants at 120 days postinoculation, whereas plants inoculated with only SCP buffer tested negative for infection. Sequencing of $\operatorname{cop} B$ and $r p o D$ PCR products amplified from plant DNA confirmed that the obtained sequences matched the sequences from the respective isolates originally used for inoculation of each plant. At 150 days postinoculation, isolation attempts resulted in the successful reisolation of $X f$ from two of the plants inoculated with AlmaReb2. Using DNA extracted from the reisolated $X f$ bacterial culture, PCR and sequencing of the $\operatorname{cop} B$ fragment revealed the sequence of $\operatorname{cop} B$ (including the 15-nt insert) to be the same as the $\operatorname{cop} B$ from isolate AlmaReb2 used in the original inoculation of these plants. Though BLS symptoms and $X f$-specific PCR on plant DNA demonstrated $X f$ infection of plants inoculated with AlmaReb1 and PierceMed1, reisolation attempts from plants inoculated with these isolates were not successful.

\section{Discussion}

Our findings demonstrate that isolates from both Xff and Xfm are present in naturally infected SHB blueberry plantings in southern Georgia. Based upon our genetic and greenhouse characterization of isolates from blueberry, $X f f$ isolates closely related to isolates from grapevine and muscadine are capable of causing BLS symptoms on SHB blueberry and are infecting SHB in at least two southern

Table 3. Xylella fastidiosa $(X f)$ isolates cultured from southern highbush (SHB) blueberries and characterized in this study

\begin{tabular}{lcllc}
\hline Site ID & Georgia county & SHB cultivar & Isolate & Subspecies $^{\mathbf{a}}$ \\
\hline 1 & Pierce & Meadowlark & PierceMed1 & Xff \\
7 & Bacon & Rebel & AlmaReb1 & Xff \\
7 & Bacon & Rebel & AlmaReb2 & Xff \\
7 & Bacon & Rebel & AlmaReb3 & Xfm \\
9 & Bacon & Star & AlmaStar1 & Xfm \\
9 & Bacon & Star & AlmaStar2 & Xfm \\
9 & Bacon & Star & AlmaStar3 & Xfm \\
\hline
\end{tabular}

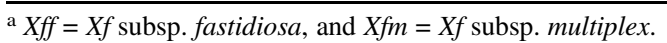


Georgia counties. To our knowledge, this represents the first report of natural infection of blueberry by Xff and also apparently represents the first time that isolates from both Xfm and Xff have been identified within a single planting of any crop in the southeastern United States, despite the fact that both subspecies are endemic within the region.

In addition to these novel findings regarding the subspecies of $X f$ in blueberry, our genetic characterization of these seven isolates from blueberry alongside the other sequenced haplotypes from other hosts represents a substantial contribution to the pool of knowledge regarding $X f$ diversity in blueberry in its own right. Prior to this work, a total of eight other $X f$ isolates from blueberry had been characterized by MLSA-E (Oliver et al. 2014; Parker et al. 2012), with only seven reported $X f$ isolates from blueberry characterized by MLST (Nunney et al. 2013), and only four $r p o D$ sequences available from blueberry

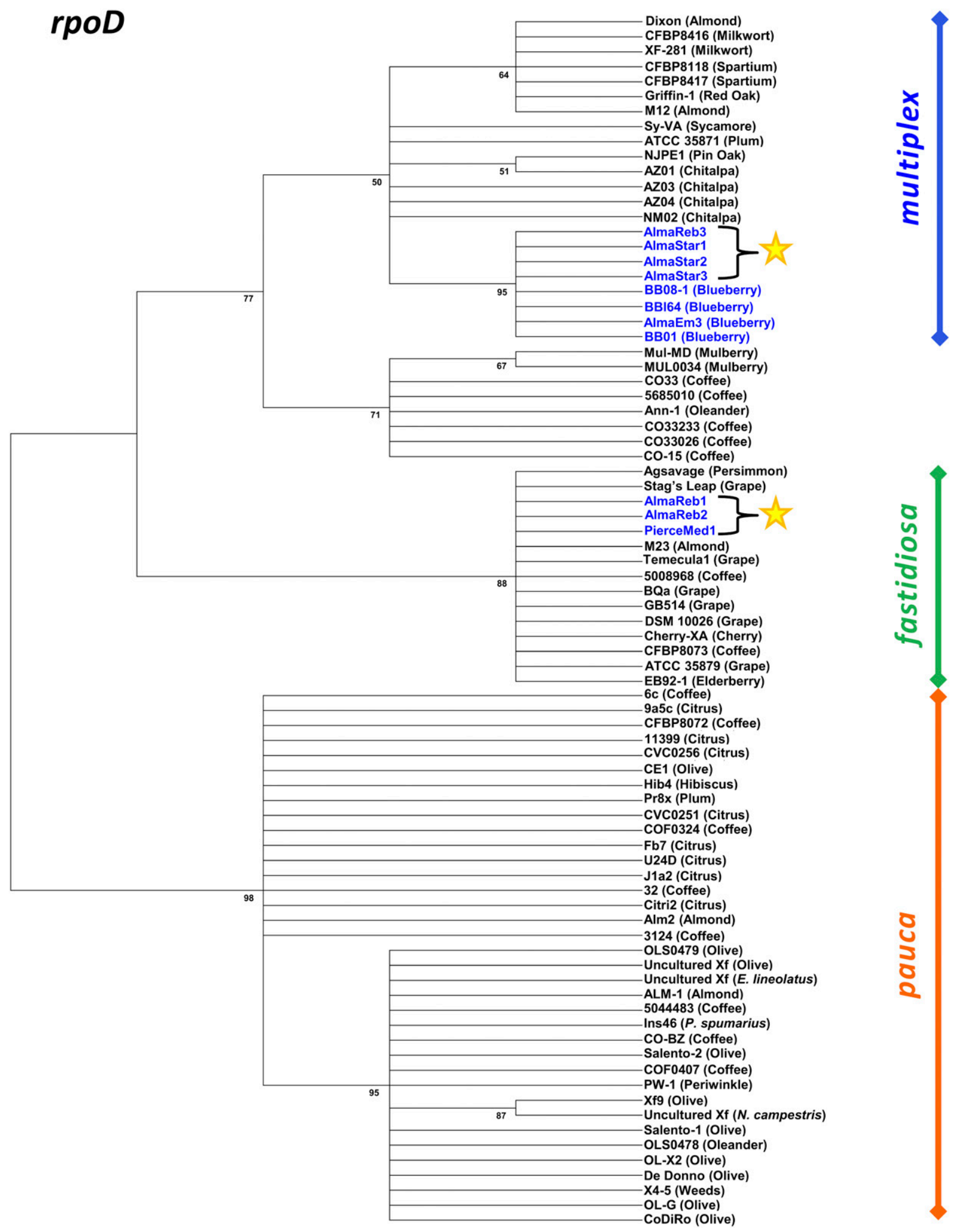

Fig. 2. rpoD neighbor-joining phylogenetic tree showing individual isolates from this study alongside Xylella fastidiosa isolates from GenBank. Common names of host of origin are given in parentheses next to each isolate name. Branches were collapsed if bootstrap support $<50 \%$. Bars to the right indicate major $X$. fastidiosa subspecies groupings. Isolates from blueberry are shown in blue (gray in print), and isolates from this work are indicated by a star. 
Xf isolates (Potnis et al. 2019; Van Horn et al. 2017). Accordingly, the three MLSA-E haplotypes reported from a single SHB planting in Bacon County, Georgia (site 7) in this study (including one previously known haplotype and two novel haplotypes) represent as many unique $X f$ haplotypes as had been sequenced previously from blueberry over the past 15 years since BLS was identified as a disease of blueberry.
The identification of new $X f$ haplotypes and $X f f$ isolates capable of naturally infecting blueberry suggests that more work is needed to understand the diversity of blueberry $X f$ isolates and the epidemiology of this disease on blueberry in the southeastern United States. The similarity between the Xff isolates characterized in this study and the Xff isolates previously characterized from grapevine and muscadine in Florida and Georgia is likely no coincidence, and it

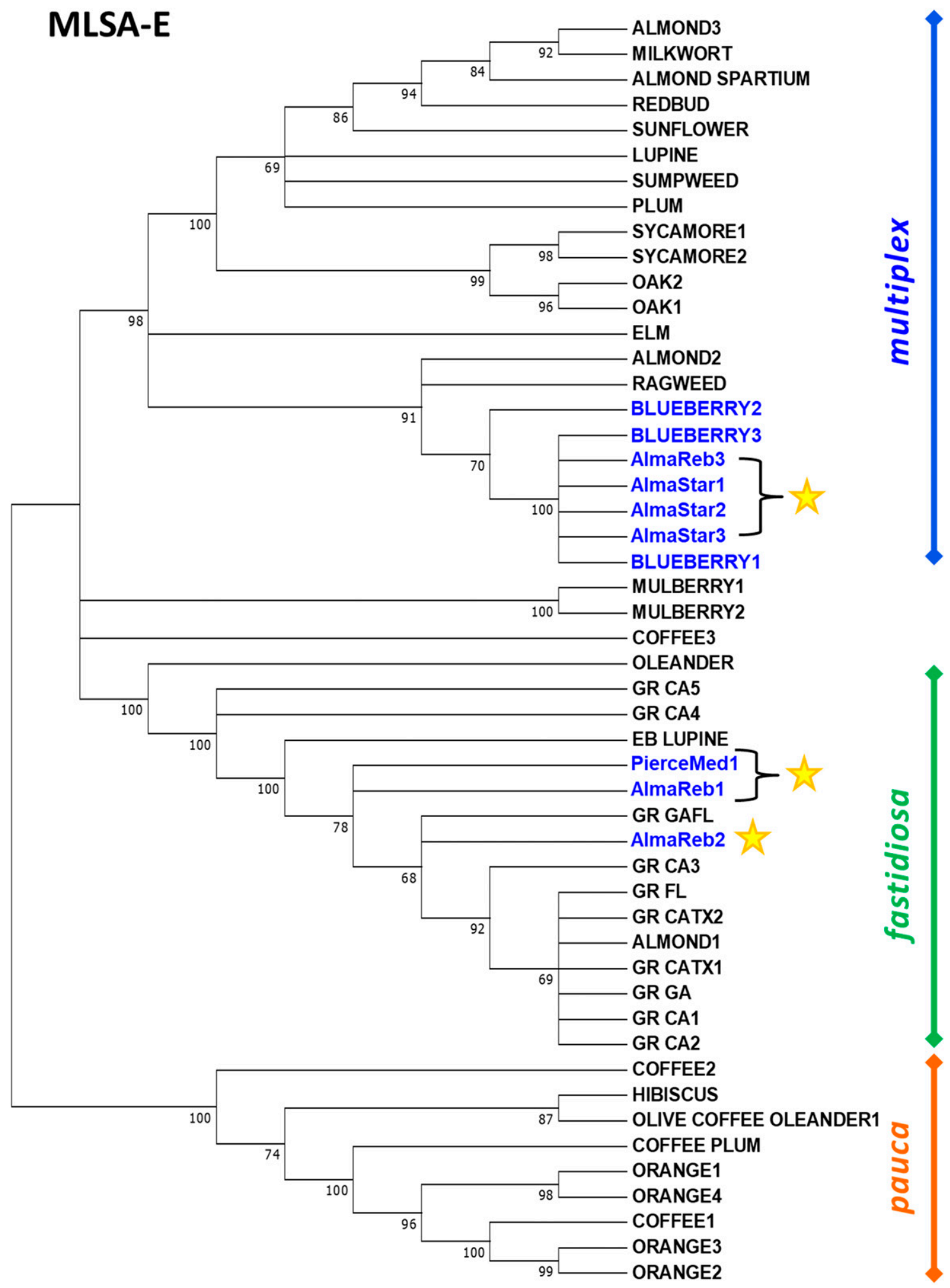

Fig. 3. Multilocus sequence analysis of environmentally mediated genes (MLSA-E) neighbor-joining phylogenetic tree showing unique Xylella fastidiosa haplotypes as well as individual isolates from this study. Branches were collapsed if bootstrap support $<50 \%$. Bars to the right indicate major $X$. fastidiosa subspecies groupings. Isolates from blueberry are shown in blue (gray in print), and isolates from this work are indicated by a star. 
suggests the possibility of movement of $X f$ between these crops. Because muscadine is a commonly grown residential and commercial crop plant in southern Georgia, and wild grape relatives are common weed species in and adjacent to blueberry plantings (including all plantings examined in this study), it is possible that these Vitis sp. may serve as reservoirs for $X f f$ isolates within blueberry plantings. To our knowledge, $X f m$ isolates have not been identified in grapevine or muscadine in the southeast, and their ability to infect Vitis sp. has not been proven. Furthermore, even though Xff isolates were found infecting SHB blueberry in two different Georgia counties in our study, the prevalence of $X f f$ isolates in blueberries remains unclear. Because the limited number of prior blueberry BLS studies did not identify Xff isolates in blueberry plantings, it may be that Xff isolates capable of infecting blueberries are relatively rare or that other barriers to transmission and infection of blueberries with $X f f$ isolates exist. It is conceivable that differences in host resistance between blueberry cultivars, differences in vector preferences between Vitis $\mathrm{sp}$. and blueberry cultivars, or differences in the ability of vector species to acquire from or transmit $X f f$ isolates between blueberry plants may make Xff isolates less capable than Xfm isolates of causing epidemics on blueberry. Along this line, using glassy-winged sharpshooters, a recent study by Burbank et al. (2019) could not demonstrate transmission of a grapevine Xff isolate despite successful acquisition from artificially inoculated SHB blueberry in controlled conditions. A prior study that used Xff isolates from grapevine for artificial inoculation of blueberries in the greenhouse showed that Xff isolates are capable of causing symptoms on SHB blueberries (Oliver et al. 2015); however, this prior study also suggested that the Xff isolates from grapevine and elderberry show significantly less virulence on blueberry relative to $X \mathrm{fm}$ isolates from blueberry. This indicates the possibility that the Xff isolates from blueberry characterized in our study may differ from the previous Xff isolates from grapevine and elderberry tested by Oliver et al. (2015) in terms of virulence on blueberry. It should be noted that although the greenhouse work carried out in fulfillment of Koch's postulates described herein was not set up to compare the virulence of $X f m$ and $X f f$ isolates, the field plants from which the Xff isolates cultured in this study were obtained showed unambiguous, severe symptoms of BLS that appeared to be as severe as symptoms on plants infected with $X f m$ isolates (data not shown). Ultimately, to determine if the Xff isolates from this study differ in virulence or pathogenicity traits from Xfm isolates from blueberry, experiments under controlled conditions need to be carried out.

The genetics underlying host specificity differences between $X f$ subspecies remains unclear, despite renewed interest in $X f$ diseases and genetic studies following the recent emergence of this pathogen in Europe. It is likely that the genetic basis of $X f$ pathogenicity is a complex trait that will not be elucidated from the characterization of the handful of genes typically utilized for multilocus sequence typing. Nonetheless, the identification and characterization of $X f$ isolates that are genetically similar yet differ in host specificity, or $X f$ isolates that are genetically distinct but are still capable of infecting a common host (such as those described here), would likely be valuable for elucidating the determinants of $X f$ pathogenicity. To this end, applying the latest genomic analysis approaches as well as $X f$ gene knockout/replacement technologies to further investigate the isolates described in this study may help us to better understand the determinants of $X f$ pathogenicity on blueberry.

In our study, isolates from both Xfm and Xff were obtained from a single planting of SHB blueberry. This has potential implications for both disease management and new $X f$ strain emergence. To

Table 4. Multilocus sequence typing results for Xylella fastidiosa isolates from site 7 in Bacon County, Georgia

\begin{tabular}{lcccccccc}
\hline Isolate & Sequence type & leuA & petC & malF & cys $G$ & holC & nuoL & glt T \\
\hline AlmaReb1 & ST1 & 1 & 1 & 1 & 1 & 1 & 1 & 1 \\
AlmaReb2 & ST1 & 1 & 1 & 1 & 1 & 1 & 1 & 1 \\
AlmaReb3 & ST42 & 6 & 3 & 5 & 12 & 4 & 3 & 3 \\
\hline
\end{tabular}

our knowledge, efforts to breed for $X f$ resistance in blueberry have not been pursued by blueberry breeders to this point, even though host resistance would be a desirable means for managing this difficult disease. Currently, there are no known SHB cultivars that are unable to be infected with $X f$; however, apparent differences in disease prevalence and severity between cultivars suggest that tolerance differences may be present within cultivated SHB varieties. Furthermore, rabbiteye blueberries appear to be infrequently affected by this disease, even when grown in close proximity to SHB that are severely impacted. It is likely that host genetic differences account for these differences in susceptibility and that these could be utilized in breeding efforts; however, differences between strains of $X f$ infecting blueberry need to be taken into account in any concerted breeding effort, because multiple haplotypes and subspecies present in a single planting could result in the failure or breakdown of resistance that is specific to a single isolate or strain. Also, as alluded to previously, it is possible that $X f$ movement between wild or cultivated Vitis sp. and blueberry may be possible, and the discovery of Xff isolates in naturally infected SHB suggests the possibility that management practices targeting infected alternate host plants or vectors may impact $X f$ infection and spread within SHB blueberries. This bears further investigation as well. For $X f$, recombination between subspecies is known to be a significant factor in adaption and movement to new host plants, and prior studies have indicated that the $X f m$ isolates previously identified from blueberry are intersubspecific recombinants between subspecies Xfm and Xff (Nunney et al. 2014). Our identification of SHB plantings with both $X f m$ and $X f f$ subspecies indicates that blueberry plantings in southern Georgia may provide opportunities for recombination between the two subspecies, which has been shown to occur artificially between $X f f$ isolates from grape and $X f m$ isolates from blueberry (Kandel et al. 2017).

As a whole, our study demonstrates that $X f$ isolates from both $X f f$ and Xfm are present in SHB plantings in southern Georgia. This confirms the suggestion of prior artificial inoculation research that isolates from both subspecies are capable of causing BLS on blueberry, and it suggests that movement of Xff from wild hosts (possibly muscadines and relatives) to cultivated blueberry may occur. Further studies regarding the epidemiological implications of these findings with respect to BLS movement on blueberry may reveal new opportunities for managing this devastating disease of blueberries in the southeastern United States.

\section{Acknowledgments}

We are grateful to Will Lovett, James Jacobs, and Joe Slusher for their help with sample collections, and to Hunter Mosley for his assistance with bacterial isolations.

\section{Literature Cited}

Almeida, R. P. P., De La Fuente, L., Koebnik, R., Lopes, J. R. S., Parnell, S., and Scherm, H. 2019. Addressing the new global threat of Xylella fastidiosa. Phytopathology 109:172-174.

Almeida, R. P. P., Nascimento, F. E., Chau, J., Prado, S. S., Tsai, C. W., Lopes, S. A., and Lopes, J. R. S. 2008. Genetic structure and biology of Xylella fastidiosa strains causing disease in citrus and coffee in Brazil. Appl. Environ. Microbiol. 74:3690-3701.

Almeida, R. P. P., and Nunney, L. 2015. How do plant diseases caused by Xylella fastidiosa emerge? Plant Dis. 99:1457-1467.

Almeida, R. P. P., and Purcell, A. H. 2003. Biological traits of Xylella fastidiosa strains from grapes and almonds. Appl. Environ. Microbiol. 69:7447-7452.

Brannen, P. M., Chang, C.-J., Boland, R. T., Horton, D. L., and Krewer, G. W. 2016. Bacterial Leaf Scorch of Blueberry. Extension Circular 922. University of Georgia, Athens, GA. https://extension.uga.edu/publications/detail.html? number=C922\&title=Bacterial\%20Leaf\%20Scorch\%20of\%20Blueberry.

Brannen, P. M., Scherm, H., and Chang, C. J. 2008. Survey of cultivar differences in bacterial leaf scorch incidence among southern highbush blueberries. Dixie Blueberry News 8:6-7.

Brlansky, R. H., Timmer, L. W., French, W. J., and Mccoy, R. E. 1983 Colonization of the sharpshooter vectors, Oncometopia nigricans and Homalodisca coagulata, by xylem-limited bacteria. Phytopathology 73: 530-535.

Burbank, L. P., Sisterson, M. S., and O'Leary, M. L. 2019. Infection of blueberry cultivar 'Emerald' with a California Pierce's disease strain of Xylella fastidiosa and acquisition by glassy-winged sharpshooter. Plant Dis. 104:154-160. 
Chang, C. J., Donaldson, R., Brannen, P., Krewer, G., and Boland, R. 2009. Bacterial leaf scorch, a new blueberry disease caused by Xylella fastidiosa. HortScience 44:413-417.

Chatterjee, S., Almeida, R. P. P., and Lindow, S. 2008. Living in two worlds: The plant and insect lifestyles of Xylella fastidiosa. Annu. Rev. Phytopathol. 46: 243-271.

Davis, M. J., French, W. J., and Schaad, N. W. 1981. Axenic culture of the bacteria associated with phony disease of peach and plum leaf scald. Curr. Microbiol. 6: 309-314.

Davis, M. J., Purcell, A. H., and Thomson, S. V. 1980. Isolation media for the Pierce's disease bacterium. Phytopathology 70:425-429.

De La Fuente, L., Parker, J. K., Oliver, J. E., Granger, S., Brannen, P. M., van Santen, E., and Cobine, P. A. 2013. The bacterial pathogen Xylella fastidiosa affects the leaf ionome of plant hosts during infection. PLoS One 8:e62945.

Doyle, J., and Doyle, J. L. 1987. A rapid DNA isolation procedure for small quantities of fresh leaf tissue. Phytochem. Bull. 19:11-15.

Ferguson, M. H., Clark, C. A., and Smith, B. J. 2017. Association of Xylella fastidiosa with yield loss and altered fruit quality in a naturally infected rabbiteye blueberry orchard. HortScience 52:1073-1079.

Francis, M., Lin, H., Cabrera-La Rosa, J., Doddapaneni, H., and Civerolo, E. L. 2006. Genome-based PCR primers for specific and sensitive detection and quantification of Xylella fastidiosa. Eur. J. Plant Pathol. 115:203-213.

Galtier, N., Gouy, M., and Gautier, C. 1996. SEAVIEW and PHYLO_WIN: Two graphic tools for sequence alignment and molecular phylogeny. Comput. Appl. Biosci. 12:543-548.

Gouy, M., Guindon, S., and Gascuel, O. 2010. SeaView version 4: A multiplatform graphical user interface for sequence alignment and phylogenetic tree building. Mol. Biol. Evol. 27:221-224.

Harmon, P. F., and Hopkins, D. L. 2009. First report of bacterial leaf scorch caused by Xylella fastidiosa on southern highbush blueberry in Florida. Plant Dis. 93:1220.

Hopkins, D., Harmon, P., and Brannen, P. 2012. Host range of Xylella fastidiosa strains that cause blueberry leaf scorch. Phytopathology 102:S4.55.

Hopkins, D. L., and Purcell, A. H. 2002. Xylella fastidiosa: Cause of Pierce's disease of grapevine and other emergent diseases. Plant Dis. 86:1056-1066.

Janse, J. D., and Obradovic, A. 2010. Xylella fastidiosa: Its biology, diagnosis, control and risks. J. Plant Pathol. 92:S35-S48.

Jolley, K. A., Chan, M. S., and Maiden, M. C. J. 2004. mlstdbNet - distributed multi-locus sequence typing (MLST) databases. BMC Bioinformatics 5:86.

Kandel, P. P., Almeida, R. P. P., Cobine, P. A., and De La Fuente, L. 2017. Natural competence rates are variable among Xylella fastidiosa strains and homologous recombination occurs in vitro between subspecies fastidiosa and multiplex. Mol. Plant-Microbe Interact. 30:589-600.

Killiny, N., and Almeida, R. P. P. 2011. Gene regulation mediates host specificity of a bacterial pathogen. Environ. Microbiol. Rep. 3:791-797.

Kumar, S., Stecher, G., and Tamura, K. 2016. MEGA7: Molecular Evolutionary Genetics Analysis version 7.0 for bigger datasets. Mol. Biol. Evol. 33: 1870-1874.

Loconsole, G., Potere, O., Boscia, D., Altamura, G., Djelouah, K., Elbeaino, T., Frasheri, D., Lorusso, D., Palmisano, F., Pollastro, P., Silletti, M. R., Trisciuzzi, N., Valentini, F., Savino, V., and Saponari, M. 2014. Detection of Xylella fastidiosa in olive trees by molecular and serological methods. J. Plant Pathol. 96:7-14.

Minsavage, G. V., Thompson, C. M., Hopkins, D. L., Leite, R. M. V. B. C., and Stall, R. E. 1994. Development of a polymerase chain reaction protocol for detection of Xylella fastidiosa in plant tissue. Phytopathology 84:456-461.

NeSmith, D. S. 2008. 'Rebel' southern highbush blueberry. HortScience 43: 1592-1593.
Nunney, L., Azad, H., and Stouthamer, R. 2019. An experimental test of the hostplant range of nonrecombinant strains of North American Xylella fastidiosa subsp. multiplex. Phytopathology 109:294-300.

Nunney, L., Hopkins, D. L., Morano, L. D., Russell, S. E., and Stouthamer, R. 2014. Intersubspecific recombination in Xylella fastidiosa strains native to the United States: Infection of novel hosts associated with an unsuccessful invasion. Appl. Environ. Microbiol. 80:1159-1169.

Nunney, L., Vickerman, D. B., Bromley, R. E., Russell, S. A., Hartman, J. R., Morano, L. D., and Stouthamer, R. 2013. Recent evolutionary radiation and host plant specialization in the Xylella fastidiosa subspecies native to the United States. Appl. Environ. Microbiol. 79:2189-2200.

Oliver, J. E., Cobine, P. A., and De La Fuente, L. 2015. Xylella fastidiosa isolates from both subsp. multiplex and fastidiosa cause disease on southern highbush blueberry (Vaccinium sp.) under greenhouse conditions. Phytopathology 105: 855-862.

Oliver, J. E., Sefick, S. A., Parker, J. K., Arnold, T., Cobine, P. A., and De La Fuente, L. 2014. Ionome changes in Xylella fastidiosa-infected Nicotiana tabacum correlate with virulence and discriminate between subspecies of bacterial isolates. Mol. Plant-Microbe Interact. 27:1048-1058.

Parker, J. K., Havird, J. C., and De La Fuente, L. 2012. Differentiation of Xylella fastidiosa strains via multilocus sequence analysis of environmentally mediated genes (MLSA-E). Appl. Environ. Microbiol. 78:1385-1396.

Potnis, N., Kandel, P. P., Merfa, M. V., Retchless, A. C., Parker, J. K., Stenger D. C., Almeida, R. P. P., Bergsma-Vlami, M., Westenberg, M., Cobine, P. A., and De La Fuente, L. 2019. Patterns of inter- and intrasubspecific homologous recombination inform eco-evolutionary dynamics of Xylella fastidiosa. ISME J. 13:2319-2333.

Purcell, A. 2013. Paradigms: Examples from the bacterium Xylella fastidiosa. Annu. Rev. Phytopathol. 51:339-356.

Purcell, A. H., and Finlay, A. 1979. Evidence for non-circulative transmission of Pierce's disease bacterium by sharpshooter leafhoppers. Phytopathology 69: 393-395.

Purcell, A. H., Finlay, A. H., and Mclean, D. L. 1979. Pierce's disease bacterium: Mechanism of transmission by leafhopper vectors. Science 206:839-841.

Purcell, A. H., and Saunders, S. R. 1999. Fate of Pierce's disease strains of Xylella fastidiosa in common riparian plants in California. Plant Dis. 83:825-830.

Saitou, N., and Nei, M. 1987. The neighbor-joining method: A new method for reconstructing phylogenetic trees. Mol. Biol. Evol. 4:406-425.

Scally, M., Schuenzel, E. L., Stouthamer, R., and Nunney, L. 2005. Multilocus sequence type system for the plant pathogen Xylella fastidiosa and relative contributions of recombination and point mutation to clonal diversity. Appl. Environ. Microbiol. 71:8491-8499.

Schaad, N. W., Postnikova, E., Lacy, G., Fatmi, M., and Chang, C. J. 2004. Xylella fastidiosa subspecies: $X$. fastidiosa subsp. piercei, subsp. nov., X. fastidiosa subsp. multiplex subsp. nov., and X. fastidiosa subsp. pauca subsp. nov. Syst. Appl. Microbiol. 27:763-763.

Tertuliano, M., Srinivasan, R., and Scherm, H. 2012. Settling behavior of the glassy-winged sharpshooter, Homalodisca vitripennis, vector of Xylella fastidiosa, on southern highbush blueberry cultivars. Entomol. Exp. Appl. 143:67-73.

Van Horn, C., Chang, C. J., and Chen, J. C. 2017. De novo whole-genome sequence of Xylella fastidiosa subsp. multiplex strain BB01 isolated from a blueberry in Georgia, USA. Genome Announc. 5:e01598-16.

Yuan, X., Morano, L., Bromley, R., Spring-Pearson, S., Stouthamer, R., and Nunney, L. 2010. Multilocus sequence typing of Xylella fastidiosa causing Pierce's disease and oleander leaf scorch in the United States. Phytopathology 100:601-611. 